\title{
The establishment and application of synthetically stiffness knowledge model of the steering system
}

\author{
Xufeng Wang
}

Automotive Engineering institute, Jiangxi University of Technology, Nanchang 330098, China

Keywords: Synthetically stiffness; Steering system; Knowledge model

\begin{abstract}
The components of the steering system, especially some bars and scaffolds, have certain elasticity, which makes the actual steering angle of steering wheel be smaller than the angle that is converted according to the transmission ratio of the steering angle when the driver turns the steering wheel, so there will be the trend of insufficient steering. Based on the analysis of the vehicle dynamic model of multi-degree of freedom, a calculation model for the stiffness of the steering system is established in the paper, which is used to calculate the design of early development, enabling the designer to exclude those schemes that are farther from the optimal results in the preliminary design of screening to reduce developmental risks.
\end{abstract}

\section{Introduction}

With the rapid development of automobile industry in the world, the car has become an important and indispensable means of transportation in people's daily life and in the industrial and agricultural production. With the popularity of cars, people's demands for cars has become increasingly high. When obtaining good power and economy, at the same time, they also require the vehicles to have good handling stabilities. The handling stability of the vehicles on the one hand determines the convenient operation for the driver. On the other hand, it also decides the driving safety of the automobile. The bad handling stability of the vehicle results in fatal car accidents that have happened many times in the world. The improvement of the handling stability of the automobile can only be realized by conducting in-depth research on the vehicle system dynamics.

With rapid and steady growth of China's economy, highway mileage is increasing constantly and freight industry develops quickly. The competition in truck manufacturing industry has become increasingly fierce, with the big truck manufacturers both at home and abroad participating in, especially the high-speed and heavy-duty trucks. Domestic enterprises in the overloaded tractor with trailer accumulate less experience, generally through the introduction of technology or joint venture to compensate for the lack of technology to obtain opportunities to participate in the competition, otherwise they would be caught in the similar weird cycle of backward technology and then introducing technology to the cars. But compared to the car, the gap between the advanced level of international technology and the design technology of the truck in China is relatively smaller, in which case digestion and absorption as well as innovation is possible.

The handling stability of the vehicle refers to the situation where in the case of drivers do not feeling much nervous and exhausted, the car can follow drivers to drive in the direction given by steering wheel through the steering system, and when encountering outside interference, the vehicle can resist the interference and keep stable driving. It does not only affect the vehicle's handling 
convenience degree, but also is a major performance to determine the safe driving of the high-speed vehicle, known as the "lifeline" of high-speed vehicles. With the continuous improvement of the road conditions, vehicle speed is greater than, and some cars even exceed. The handling stability of the vehicle is paid more and more attention to, becoming one of the important properties of modern automobile. A lot of cars have the problem of "high speed floating" ---- at a high speed drivers often feel difficult to control the direction of the vehicle. But as for some cars, although the speed is very high, drivers are confident to accurately control the running direction of the vehicle. For average motors, the higher the speed is, the more prominent the handling stability problems will be and the more serious the accidents they cause will be. However, this does not mean that the low-speed car has no handling stability problem. Actually there are some cases of some trucks, jeeps and wagons which cause accidents because of losing control in direction, mostly drifting.

The components of the steering system, especially some bars and scaffolds, have certain elasticity, which makes the actual steering angle of steering wheel smaller than the angle that is converted according to the transmission ratio of the steering angle when the driver turns the steering wheel, so there will be the trend of insufficient steering. The steering system synthetical stiffness is one of the parameters required to input when modeling the computational mechanics for handling stability, including steering column, fixed bracket of the steering gear, the stiffness of driving rod, which have an effect on the handling stability of the whole vehicle. For example, insufficient steering system synthetical stiffness causes the swinging and floating when the vehicle is driving at a high speed. The steering system synthetical stiffness also affects the cornering stiffness of the tire greatly.

The vehicle suspension can be divided into independent suspension and non independent suspension. As for the non independent suspension vehicle, because its axle is integrated, when the supporting axle makes the wheels off the ground, the structural relationship between the various parts of the steering system remains unchanged, so the steering system stiffness can be measured in this working condition. As for the vehicle with independent suspension, when the chassis is supported, the position of the wheel and the steering system will deviate greatly from their working position, making the measured parameters of no practical significance, so vehicle with independent suspension must place the wheel on the floating disc so as to measure effectively the steering system stiffness. In addition, for the vehicle with independent suspension, because the vertical swing of the suspension lower arm will cause the longitudinal displacement of the steering wheel and the steering compliance, when the steering system stiffness is measured, the measurement of the vertical swing stiffness of the lower arm of the suspension and the vertical swing angle stiffness should also be involved. The suspension-steering system of independent suspension vehicle is more complex than that of non-independent one, which brings more difficulties to the actual measurement.

Based on the analysis of the vehicle dynamic model of multi-degree of freedom, a calculation model for the stiffness of the steering system is established in the paper, which is used to calculate the design of early development, enabling the designer to exclude those schemes that are farther from the optimal results in the preliminary design of screening to reduce developmental risks. Then, based on synthetical rigidity calculation model of the steering system, the paper establishes several experimental models to measure the steering system synthetical stiffness. After comparison, the best test method is determined, providing a test method of the practical value and guidance for the trial after the product design work. 


\section{Methods}

The handling stability of the vehicle refers to the situation where in the case of the driver not feeling too nervous and exhausted, the car can follow the driver to drive in the direction given by steering wheel through the steering system, and when encountering outside interference, the vehicle can resist the interference and keep stable driving. Handling stability is one of the important essential properties of the vehicle.

The systematic research of vehicle handling stability started as early as forty years ago, and has made many valuable research results, which makes the modern car more sophisticated in operation stability than the early automobile. There are plentiful related research literature, but there is still lack of explicit understanding in many aspects. Now, the research of computer simulation can simulate all the details of the vehicle handling characteristics more and more specifically, but some basic problems remain unsolved fundamentally. For example, how to evaluate exactly the vehicle handling stability performance from theory and experiment is still not clear. If the evaluation standard of vehicle handling stability is fuzzy, it will encounter fundamental obstacles in research on how to obtain good performance.

If a clear understanding of evaluation standard can be got, it will make the research of vehicle handling stability step forward. In order to promote the understanding of this issue, in recent years, there is an interesting research direction; namely, regarding the car and the driver as a closed-loop system to study. At the same time, studies on the dynamic characteristics of the driver will become more and more important. Of course, the research on the closed-loop system, after all, is to obtain automobiles with good characteristics. Therefore, the research on the closed-loop system is not to deny the open-loop system which is the research method that only studies the characteristics of the vehicle itself but to provide a more reliable premise. In a sense, it is to propose more exact evaluation indicators for car characteristics. The research results of evaluation indicators tend to produce new requirements on test methods and means.

From the previous section of the discussion, the vehicle dynamics model is the core part of the simulation and the results of the simulation depend on the vehicle dynamics model. The vehicle dynamics model ranges from the simplest two degree of freedom model to complex eleven degree of freedom model. The complex dynamic model can get more accurate simulation results, but it requires more input parameters. With scholars' efforts, the mechanical model gradually develops from the linear model to nonlinear lumped parameter model and the degree of freedom of the model develops from two degrees to more than ten degrees. The representative one is the fifteen degree of freedom model established by, seventeen degrees of freedom model established by University of Michigan, USA, eighteen degree of freedom model established by. The fifteen degrees of freedom are spring mass, six moves along three coordinate axes and the front steering wheel rotating around three axes, five beats, and pin deflection, rotation around the axis of rotation of the rear wheel, four beats, rotating around the axis of rotation.

If the steering system stiffness is decomposed, it can be understood that it consists of the following three parts of rigidity:

1. The elasticity above the steering gear, mainly the steering column, its stiffness is,

2. The elasticity of the connecting rod and the hinge point between the steering gear and steering wheel; its stiffness is

3. The connecting elasticity of the components connected with the body and frame, mainly the fixed stiffness of the steering gear; its stiffness is $\mathrm{C}_{3}$. 
The stiffness model of steering system is shown in Fig. 1.

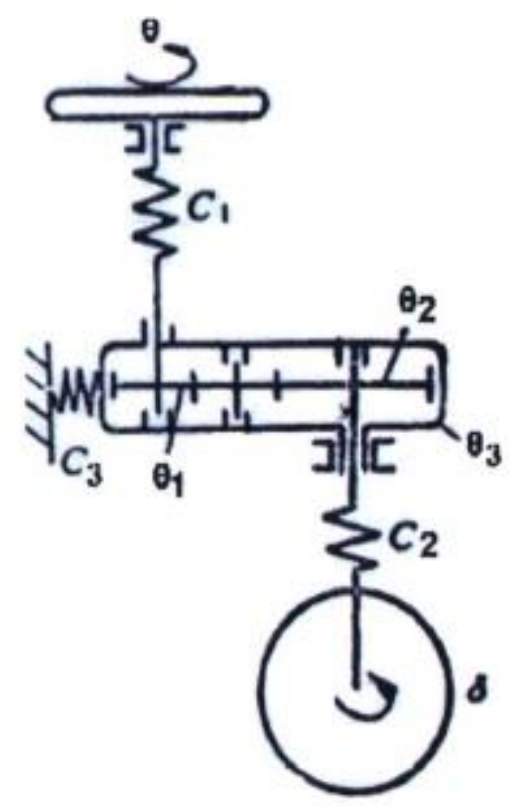

Fig. 1 the stiffness model of steering system

The development of science and technology and the test technology are closely linked. Modern natural science is formed after the experimental science appeared. Many important scientific results are obtained by means of the new test, so the possession of the scientific advanced testing technique is an important symbol of the modernization of science and technology. So it is with the auto industry.

The characteristics of the automobile industry are large production, various products and complex conditions to use the products, which requires more about the performance, service life, quality, cost and others of the products. There are numerous factors affecting the quality of products and it involves many technical fields, so the study of some problems is not enough. Therefore, the design of new automobile products or the existing products, even though the design and manufacture have been considered very carefully, must pass a test to be examined. The test is to check whether the design idea is correct, whether the design intention can be implemented and whether the design product is suitable for use. The car test can help people understand profoundly the essence and rules in the various phenomena in the actual use, explore the solution to the existing problems and verify the effect and degree of the solution, which is a vital method to promote the progress of the automobile technology as well as an important means to ensure the performance of the product and improve the quality and competitiveness of the product. Automobile test is a process where the some parameters in the tested system are motivated artificially through a special device and are tested by universal or special instrument which analyzes the data.

Based on the theoretical calculation model for the steering system synthetical stiffness illustrated above, many factors compose the stiffness. In order to provide input parameters more accurately for the vehicle dynamics model, we can obtain accurate steering system synthetical stiffness value more intuitively through experiment tests, thus inputting precise parameters to the vehicle dynamics model, which can avoid or reduce the discrete of the results caused by some numerical errors or incorrectness. 
Analyzing from the purpose of the experiment and the means that is accurate test data and test equipment investment taken to achieve the purpose, with heavy commercial vehicles as the research field, integrating the advantages of various methods, we establish a more practical test method which is shown in Fig. 1. In the method, the steering wheel is fixed and the front axle is supported with two support blocks and fixed with bolts. To make the two front wheels off the ground the reinforcing bar is installed on the left wheel. With steel wire rope going through the fixed pulley and removing code in the steering system, the reinforcing bar generates tension, so that the angular displacement of the wheel steering can be measured by a vertical measuring instrument. Specifically, every time the wheel turns to a position, the vertical instrument is used to cast shadows on the ground for the front and rear wheel and the two points, which requires a person to assist to get a straight line, and then the angle between this line and the original location is directly measured to obtain the wheel rotation angle. The steering system is loaded to generate five times left and right turns each. Every time it is load, a group of data will be tested in the process.

The wheel rotates around the main pin under the effect of load. The balance torque of the steering system in the main pin is:

$$
\mathrm{M}=\mathrm{P} * \mathrm{~L}
$$

In order to verify the test model in the previous chapter, I choose a $4 X 2$ heavy-duty tractor of a company. The third test method and the best scheme are used to conduct the actual test respectively and the results are to be analyzed.

The target value of the steering system integrated stiffness design of the selected model is $1020 \mathrm{Nm} / \mathrm{deg}$.

Comparing the two test results, we can see that the steering system synthetical stiffness measured by the best test method is closer to the design target value than the value measured by the third method. The reason is that the final scheme eliminates the effect of rim strength and local stiffness, thus improving the accuracy.

It is worth noting that in order to eliminate the gap error of the test system, the initial record point in the scheme is when loading $1000 \mathrm{~N}$. Therefore, from the above analysis, we can basically determine that the testing method is feasible.

\section{Conclusion}

At present, the domestic heavy commercial vehicle market develops from the exclusive Ji'nan Heavy Truck Corporation in the past three years to the present Ji'nan Heavy Truck, Foton, FAW, Shaanxi Auto and others, coupled with the joint venture of the world heavy truck giants and Ji'nan heavy truck to produce high-grade HOWO heavy commercial vehicles. Recently, Sichuan Auto with SAIC Group Holdings and Italian company intend to work together to produce branded heavy commercial vehicle in our country. Various phenomena indicate that the current domestic demand of the heavy commercial market is still rising. With high-end international brands entering China and producing products in the domestic market, the technology of those products is completely synchronized with the world or the gap gets smaller, but the price is far lower than the fully-imported cars. The original high-end products that completely rely on imports will be gradually replaced because of the price. Then, how do these domestic manufacturers make their products for some space in the market? From what China's automobiles have gone through in many years, especially the cars, only through technical cooperation, optimizing grafting, and constantly improving its own the technological 
content and technical level, coupled with China's cheap labor and low material costs, can China's automobiles widen the gap between the imported vehicles in price and get close to them in the technological level, so as to really obtain the space for survival and development. Otherwise, they would end up with the fate of being eliminated from the market.

As one of the core technology of heavy commercial vehicles, handling stability is particularly important in this war. The foreign well-known heavy vehicles apply the various advanced techniques, whose ride comfort is even better than luxury cars. In this aspect the domestic vehicles obviously fall behind, which mostly apply the Steyr, the technology in the 1970s. Except the dump truck in the construction site, with the continuous improvement of the domestic road, the driver requires more about the driving comfort. Therefore, objectively the requirement of how to improve the vehicle's handling stability is raised for the host plant or research units. Because the foundation of the heavy vehicle is weak, the investment cost of the research on heavy vehicles is high and many related test equipment can not be used for heavy vehicles, the domestic research is still at the initial stage.

Now, some domestic heavy vehicle manufacturers, after years of dormancy, put a lot of resources in the research and manufacturing of high-end heavy commercial vehicle. They spare no expense in introducing technology and talents. The range of the theoretical knowledge of the domestic heavy vehicle is vast. How to form the research results of the driver-vehicle-environment closed-loop system in the relevant review in this paper remains to be discussed further.

To be engaged in the study of operation stability must be perseverant, starting from the foundation, and step by step, some significant can be achieved.

\section{Acknowledgements}

This work was financially supported by the key subject building project (vehicle engineering) of Jiangxi University of Technology.

\section{References}

[1] Zhang J, Zhang Y, Chen L, et al. A fuzzy control strategy and optimization for four wheel steering system $[\mathrm{C}] / /$ Vehicular Electronics and Safety, 2007. ICVES. IEEE International Conference on. IEEE, 2007: 1-6.

[2] Ma Y, Xie S, Zhang X, et al. Hybrid modeling approach for vehicle frame coupled with nonlinear dampers $[\mathrm{J}]$. Communications in Nonlinear Science and Numerical Simulation, 2013, 18(4): 1079-1094.

[3] Shi Y, Wang M. Discussion of Educational Reform to the Course of "Multimedia Technology and Application" $[\mathrm{M}] / /$ Advances in Computer Science, Environment, Ecoinformatics, and Education. Springer Berlin Heidelberg, 2011: 303-307.

[4] Rohatgi P K. Low-cost, fly-ash-containing aluminum-matrix composites[J]. JOM, 1994, 46(11): 55-59.

[5] DELLE MACCHINE M E C. PARALLEL ROBOTIC SYSTEM DESIGN FOR THE STEERING AND GUIDANCE MECHANISM OF AN AUTONOMOUS UNDERWATER VEHICLE[J]. 
[6] Systemic Design Methodologies for Electrical Energy Systems: Analysis, Synthesis and Management[M]. John Wiley \& Sons, 2012.

[7] Gopalakrishnan K, Khaitan S K. Development of an intelligent pavement analysis toolbox[J]. Proceedings of the ICE-Transport, 2010, 163(4): 211-221.

[8] Norgren L, Nilsson R, Perez E, et al. Needs-driven R\&D programmes in sectorial innovation systems[J]. VINNOVA, Verket för Innovationssystem, Swedish Governmental Agency for Innovation Systems, Stockholm, 2007.

[9] Hale C, Ching C, Brett B, et al. Survey of human systems integration (HSI) tools for USCG acquisitions[R]. COAST GUARD RESEARCH AND DEVELOPMENT CENTER NEW LONDON CT, 2009.

[10]McCarthy M. Development of a generic assessment methodology for advanced safety systems[J]. APPROSYS, Washington, DC, 2009. 